(C) (C) 2014 IEEE. Personal use of this material is permitted. Permission from IEEE must be obtained for all other uses, in any current or future media, including reprinting/republishing this material for advertising or promotional purposes, creating new collective works, for resale or redistribution to servers or lists, or reuse of any copyrighted component of this work in other works. 


\title{
Design and Control of Needle Positioner for MRI-guided Laser Ablation of the Liver
}

\author{
E. Franco, M. Ristic \\ Mechanical Engineering Department, Imperial College London \\ London, UK \\ ef1311@imperial.ac.uk
}

\begin{abstract}
This paper presents the design and control of a pneumatic needle positioner for laser ablation of liver tumors under guidance by Magnetic Resonance Imaging (MRI). The prototype was developed to provide accurate point-to-point remote positioning of a needle guide inside an MR scanner with the aim of evaluating the potential advantages over the manual procedure. In order to minimize alterations to the MR environment the system employs plastic pneumatic actuators and 9 m long supply lines connecting with the control hardware located outside the magnet room. An optimized Sliding Mode Control (SMC) scheme was designed for the position control of the device. Wireless micro-coil fiducials are used for automatic registration in the reference frame of the MR scanner. The MRIcompatibility and the accuracy of the prototype are demonstrated with experiments in the MR scanner.
\end{abstract}

Keywords-Medical Robotics; Pneumatic Systems; MRI.

\section{INTRODUCTION}

Laser Interstitial Thermoablation (LITT) is used to treat otherwise inoperable liver tumors by delivering thermal energy from a high power laser through a catheter probe directly to the target lesions [1]. Compared to ultrasound guidance the MRIguided procedure offers better soft tissue contrast and provides the ability to monitor the tissue temperature rise and to control the therapy delivery [2]. Open MR magnets have been used for this procedure to target the lesions with a coaxial puncture needle and to undertake thermal monitoring [3]. However the lower field and gradient strength of this type of scanners limit their imaging capability and speed. Cylindrical magnet MR scanners are commonly available in most hospitals, but the patient access is severely restricted. Consequently it is usual to have the catheter probe placed under ultrasound guidance at a different site in the department and to move then the patient to the MR scanner [4]. This approach results in a more complex and time consuming workflow. Moving the patient after the catheter insertion also bears the risk of dislodging the catheter probe compromising targeting accuracy.

The aim of this research is the development of an actuated mechatronic needle positioner that will allow accurate alignment of a suitable needle guide while the patient is in the MR scanner and will assist manual needle insertion outside the bore with the goal of reducing procedure times and improving targeting accuracy. This paper presents the design and control of a first prototype with 3 degrees-of-freedom (DOF), low pressure pneumatic actuation and long supply lines.

A needle-guiding robot for microwave thermotherapy of liver tumors was developed by Hata et al [5]. The device employs ultrasonic motors which resulted in noticeable image degradation when actuated inside the MRI scanner. Recently pneumatic actuation has been chosen for several MRIcompatible robotic devices because it produces minimum alterations to the MR environment. Song et al [6] developed a 4-DOF robot for transperineal prostate biopsy with pneumatic actuation and $5 \mathrm{~m}$ supply lines. Melzer et al [7] proposed a 6DOF MRI-compatible robot actuated by special low-friction pneumatic cylinders with the control unit residing in the MRI scanner room. Yang et al [8] developed a 4-DOF parallel robot for breast biopsy actuated by a piezo motor and by pneumatic cylinders with long supply lines. In spite of keeping the control unit outside the scanner room operating the device in the bore led to noticeable SNR reduction due to metallic parts in pneumatic cylinders and to the piezo motor.

The prototype presented in this paper is actuated by commercially available plastic pneumatic cylinders. The use of low actuating pressure enhances safety and reduces air consumption. In order to minimize alterations to the MR environment all control hardware including the pneumatic valves is kept outside the magnet room thanks to the use of $9 \mathrm{~m}$ long supply lines. Position errors below $1 \mathrm{~mm}$ were achieved in [9] for a similar pneumatic system using SMC schemes. This paper presents an optimized SMC scheme that achieved steadystate position errors below $0.15 \mathrm{~mm}$ and at the same time resulted in lower overshoot and shorter settling time compared to a standard SMC design. In order to enhance the accuracy of the needle positioner in the MR scanner wireless micro-coil fiducials attached to the needle guide are used for initial registration. The position of the fiducials is calculated with a tracking algorithm that provides sub-millimeter accuracy [10].

This paper is organized as follows. Section II presents the design requirements. Section III outlines the system development. Section IV presents the controller design. Section $\mathrm{V}$ reports experimental results and Section VI contains concluding remarks. 

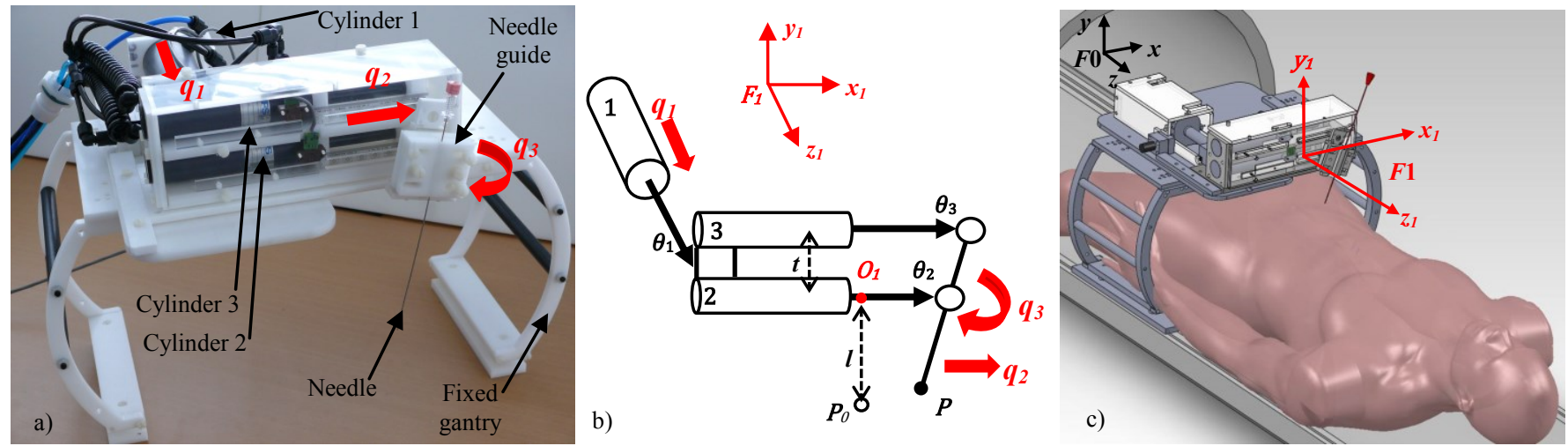

Fig. 1. Prototype of the needle positioner (a); kinematic diagram in isometric view: $q_{1}, q_{2}, q_{3}$ are the actuated movements of the needle guide, $\theta_{1}, \theta_{2}, \theta_{3}$ are the measured piston positions, cylinders are numbered form 1 to 3 (b); CAD model: $F 1$ and $F 0$ are the coordinate frames of positioner and MRI scanner (c).

\section{SYSTEM REQUIREMENTS}

In LITT procedures the patient lays supine with an MR receiver coil on his abdomen. The needle positioner should operate in the scanner bore consequently it should be MRI-safe and it should not degrade the quality of the MR images. The average size of the liver in adults is $21 \mathrm{~cm}$ across its widest point, $16 \mathrm{~cm}$ at its greatest vertical height and $11 \mathrm{~cm}$ from back to back [11]. The lesions normally treated vary in size from 10 $\mathrm{mm}$ to $50 \mathrm{~mm}$ and they can be located in any part of the liver [1]. The workspace of the device should cover an area of $160 \times 210 \mathrm{~mm}$ on a coronal $(x z)$ plane. Typically the needle can be inserted inclined on both axial (xy) and sagittal $(z y)$ planes. Consequently the needle guide should be capable of two translations and two rotations. Based on the typical size of lesions $3 \mathrm{~mm}$ targeting accuracy is considered sufficient. The MR image resolution varies between $0.8 \mathrm{~mm}$ and $1.5 \mathrm{~mm}$ and targeting accuracy comparable to the pixel size is desired. The needle positioner is required to carry out point-to-point positioning tasks moving in a slow and controlled fashion.

\section{SYSTEM DESIGN}

The prototype presented in this paper is intended to provide accurate point-to-point positioning of a needle guide inside a closed bore MR scanner and to assist the manual needle insertion outside the bore during phantom trials. The system consists of a remotely actuated positioner, a control unit containing control hardware and micro-controller, a graphical user interface on a host PC. This section describes the design of the prototype.

\section{A. Robot Structure}

The prototype was designed to be placed inside the MR scanner secured to the patient bed and consists of a fixed gantry and of a remotely actuated unit carrying a needle guide (Fig. 1.a). To ensure MRI compatibility plastic materials (Delrin, PTFE) were employed for the construction and double-acting plastic cylinders (IPS Inc.) were chosen for the actuation. In this first prototype the needle guide can translate along the $\boldsymbol{x}_{\boldsymbol{1}}$ and $\boldsymbol{z}_{1}$ axes (movements $q_{1}, q_{2}$ ) and can only rotate around the $\boldsymbol{z}_{1}$ axis (movement $q_{3}$ ) in the base frame $F 1$ (Fig. 1.b). While this setup constrains the insertion direction on axial planes which is representative of only a subset of liver ablation procedures a modular structure was adopted in view of the introduction of a $4^{\text {th }}$ DOF. Cylinder 1 is mounted orthogonally to the other two and moves them along the longitudinal axis of the MR scanner ( $1^{\text {st }}$ DOF: $\left.q_{1}\right)$. Cylinder 2 and Cylinder 3 are arranged to provide needle translation $\left(2^{\text {nd }} \mathrm{DOF}: q_{2}\right)$ when moved together and rotation ( $3^{\text {rd }}$ DOF: $q_{3}$ ) when moved differentially. The needle guide is attached to pistons 2 and 3 with revolute joints. The relation between the joint coordinates $\left(q_{1}, q_{2}, q_{3}\right)$ and the measured positions of the pistons $\left(\theta_{1}, \theta_{2}, \theta_{3}\right)$ is:

$$
\left\{\begin{array}{c}
q_{1}=\theta_{1} \\
q_{2}=\theta_{2} \\
q_{3}=\tan ^{-1}\left(\left(\theta_{3}-\theta_{2}\right) / t\right)
\end{array}\right.
$$

The parameter $t$ is the distance between Cylinder 2 and Cylinder 3. The base coordinate frame $F 1$ was assigned with the axes $\boldsymbol{z}_{1}$ and $\boldsymbol{x}_{\boldsymbol{1}}$ parallel to the prismatic joints $q_{1}$ and $q_{2}$ respectively. The fixed origin $O 1$ of $F 1$ is taken coincident to the lower revolute joint when all pistons are retracted. The needle tip $P$ is located at a distance $l$ from the origin $O 1$. The relation between the generic position of $P\left(q_{1}, q_{2}, q_{3}\right)$ in the frame $F 1$, its initial position $P_{0}(0,0,0)$ and the joint coordinates is:

$$
P\left(q_{1}, q_{2}, q_{3}\right)=T 1\left(q_{1}\right) \cdot T_{2}^{1} \cdot T 2\left(q_{2}\right) \cdot T_{3}^{2} \cdot T 3\left(q_{3}\right) \cdot P_{0}
$$

The terms $T 1, T 2, T 3$ are $4 \times 4$ homogeneous transformation matrices, function of the joint coordinates. The misalignment between the joints $q_{2}, q_{3}$ and the axes $\boldsymbol{x}_{1}, \boldsymbol{z}_{1}$ caused by manufacturing and assembly tolerances is accounted for by the constant matrices $T_{2}^{l}$ and $T_{3}^{2}$ that are computed during calibration. The linear range of motion of the prismatic joints $q_{1}$ and $q_{2}$ is $80 \mathrm{~mm}$. The angular range of motion of the revolute joint $q_{3}$ is $+45^{\circ}$ to $-45^{\circ}$ when $q_{2}$ is at mid-range. The limits on the workspace are dictated by the available space in the scanner bore which restricts the maximum size allowed for the actuators. Using the full range of motion allows targeting points within a volume corresponding to $60 \%$ of the liver without having to manually reposition the gantry on the patient bed. For the LITT procedure the workspace is however considered acceptable since the location of the lesions within the liver is generally known from pre-interventional MR 
TABLE I

ACTUATOR SELECTION

\begin{tabular}{|c|c|c|c|c|}
\hline Cylinder & $\begin{array}{c}\text { Friction } \\
\text { force }[\mathbf{N}]\end{array}$ & $\begin{array}{c}\text { Inertial } \\
\text { load }[\mathbf{N}]\end{array}$ & $\begin{array}{c}\text { Calculated } \\
\text { Bore }[\mathbf{m m}]\end{array}$ & $\begin{array}{c}\text { Stock bore } \\
{[\mathbf{m m}]}\end{array}$ \\
\hline 1 & 22 & 0.5 & 28 & 31.75 \\
\hline 2 & 10 & 0.1 & 16 & 19 \\
\hline 3 & 10 & 0.1 & 16 & 19 \\
\hline
\end{tabular}

Nominal piston friction corresponds to 0.35 bar pressure. Inertial loads assume maximum acceleration $200 \mathrm{~mm} / \mathrm{s}^{2}$. The piston mass is $0.3 \mathrm{~kg}$. A constant 2 bar pressure is considered acting in the cylinder front chamber. Stock bore is chosen immediately larger than calculated bore.

images. Consequently the gantry can be positioned accordingly on the patient bed at the start of the procedure (Fig. 1.c).

\section{B. Actuation and Sensing}

The size of the actuators was selected based on the space constraints in the MR scanner and on the external loads. The bore diameter of a 3T MR scanner is typically $60 \mathrm{~cm}$ to $70 \mathrm{~cm}$ (Siemens Verio) and the gantry should allow at least $25 \mathrm{~cm}$ clearance from the patient bed. Considering that Cylinder 2 and 3 are orthogonal to the axis of the bore their maximum extended length is $280 \mathrm{~mm}$. This limits the cylinder stroke to about $100 \mathrm{~mm}$ which allows sufficient clearance with the scanner. The cylinder bore was chosen considering only structural, inertial loads and friction forces (Table I) since the prototype is not intended to perform automatic needle insertion. The pneumatic circuit (Fig. 2) features a proportional valve (Tecno Basic, Hoerbiger) supplying all cylinder front chambers at 2.3 bar while individual proportional valves are used to set the pressure within the range 0.3 bar to 2.3 bar in the cylinder back chambers. This ensures motion in both directions due to the different effective piston area in each cylinder chamber. The actuators behave like single acting cylinders with the advantage that a steel spring is not required, which would adversely affect the MRI compatibility of the system. This design choice reduces the number of supply lines from 6 to 4 resulting in space saving inside the MR scanner and requires fewer proportional valves than other similar devices [6] [7]. In spite of the common supply to the front chamber no mutual coupling between actuators was observed due to the slow movement of the pistons and the large flow rate the

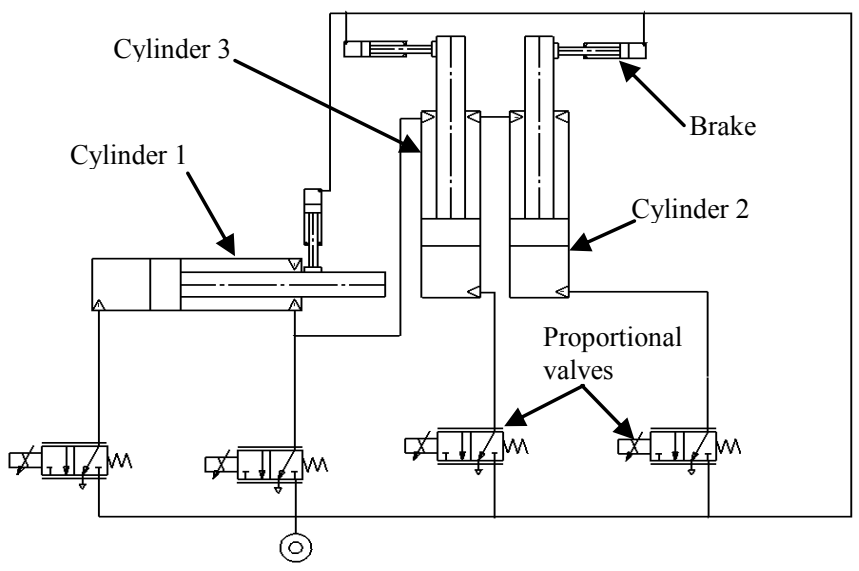

Fig. 2. Pneumatic circuit diagram.

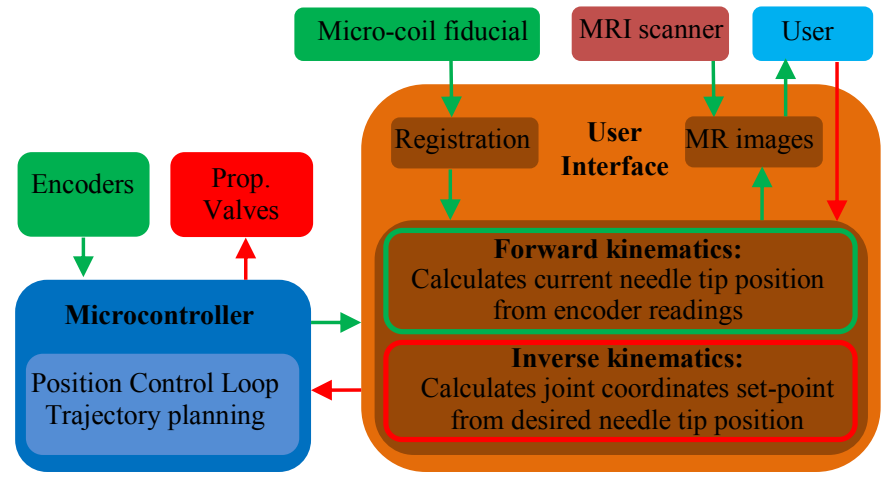

Fig. 3. Block-diagram of the system. Red arrows represent commands, green arrows represent feedback. The inner control loop runs on the microcontroller. The user closes the outer control loop using MR image feedback.

proportional valves can provide (ref. Section V). The maximum pressure in the cylinders is limited to 2.3 bar in order to enhance safety and to reduce air consumption. Non-metallic pneumatic brakes mounted orthogonally to each cylinder axis allow locking the pistons in position. Each brake consists of a miniature pneumatic cylinder with a silicone O-ring that acts as a return spring. Linear encoders (EM1-250, US Digital) and quadrature counters (LS7366, LSI Computer Systems Inc.) are employed for position sensing $(0.025 \mathrm{~mm}$ resolution).

A $9 \mathrm{~m}$ long shielded cable and $9 \mathrm{~m}$ long supply lines connect the positioner to the control unit, containing all electronic components and valves, outside the MR scanner room. An aluminum wave guide adapter with chassis-mount pi-section filters serves as interface for the cable on both sides of the panel to prevent EM noise from perturbing the MR environment. The microcontroller (mbed NXP LPC1768) in the control unit runs the real-time position control and the trajectory planning for each piston at $1 \mathrm{kHz}$. A Linux PC hosts a user interface that communicates with the microcontroller and displays the MR images after each scan (Fig. 3). The user selects the target point graphically while the computed needle tip position is displayed on the MR image for verification.

Wireless micro-coil fiducials [13] tuned at the Larmor frequency corresponding to the field strength of the MRI scanner (123.4 MHz at 2.9 T for Siemens Verio) are used for automatic coordinate registration in the MRI scanner. Each tunable micro-coil consists of a non-magnetic air core inductor (Coil Craft) filled with water gel (Spenco Healthcare Ltd) and of a ceramic trimmer capacitor in parallel with a fixed
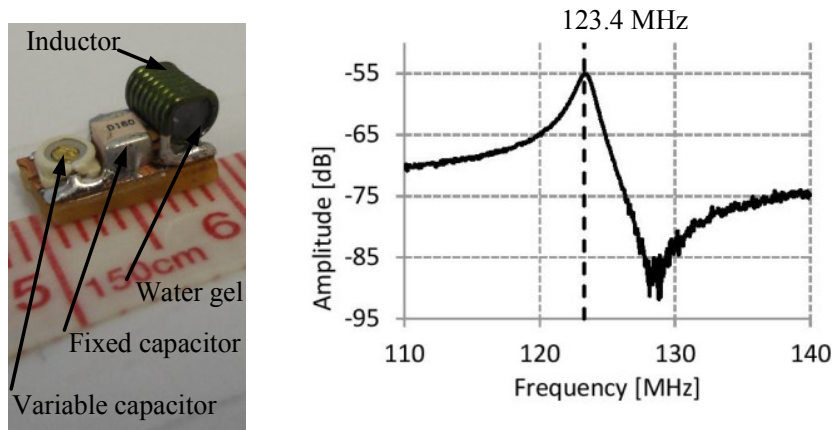

Fig. 4. Wireless micro-coil fiducial (left) and frequency response (right). 
capacitor (Passive Plus Inc.). The overall micro-coil assembly measures $10 \mathrm{~mm}$ in length and $5 \mathrm{~mm}$ in width and height (Fig. 4). The fiducials are attached directly to the needle guide and their position is measured in the reference frame $F 0$ of the MR scanner using a tracking algorithm that provides sub-millimeter accuracy [10]. This approach reduces registration errors since it locates the needle guide directly and is therefore insensitive to the manufacturing tolerances of the supporting structure.

\section{Calibration}

The prototype was calibrated using independent measurements with an optical tracking system (Optotrak Certus, NDI). An optical marker (Smart Marker, NDI) was mounted on an adapter $200 \mathrm{~mm}$ long attached to the needle guide, corresponding to the length of the coaxial needle. The identification of joint axes outlined in [12] was implemented: the actuated joints were moved one by one through their whole range of motion while the coordinates of the optical marker were independently measured with the tracking system. The constant matrices $T^{l}{ }_{2}$ and $T_{3}^{2}$, the adapter length $l$ and the distance $t$ in (1) were then estimated by least-squares from the measured points.

\section{CONTROLler DESIGN}

An optimized SMC scheme based on SMC theory [14] which includes two saturation functions was designed to control the position of the pistons. The control design is based on the reduced-order model of the piston's dynamics:

$$
A \times U-A^{\prime} \times P_{2}=m \ddot{x}+\dot{x}+F_{a} \operatorname{sign}(\dot{x})
$$

The terms $m, A, A^{\prime}, P_{2}$ are respectively the mass of the piston, the effective piston areas and the pressure in the front cylinder chamber. The terms $\gamma$ and $F_{a}$ are the actual unknown values of viscous and Coulomb friction. Similarly to [9] the much faster dynamics of the proportional valve and the delay introduced by the pipes are not modeled. To construct the control law $U$ the positive definite Lyapunov function $V=1 / 2 S^{2}$ is defined, where $S$ is a function of the piston position $x$ and velocity $\dot{x}$ and of their prescribed values [14]:

$$
S=\lambda\left(x_{S E T}-x\right)+\left(\dot{x}_{S E T}-\dot{x}\right)
$$

The parameter $\lambda=12.5 \mathrm{~Hz}$ was chosen, representing $1 / 3$ of the frequency corresponding to the largest un-modeled delay as indicated in [14]. The delay (approximately $28 \mathrm{~ms}$ ) was measured from the step response of the proportional valve connected to a pressure sensor (MPX4520GDP, $1 \mathrm{~ms}$ response time) with a $9 \mathrm{~m}$ pipe. The piston velocity $\dot{x}$ is calculated by discrete differentiation of the measured position $x$ followed by first order low pass Butterworth filter. The corner frequency of the filter was set to $120 \mathrm{~Hz}$ considering that the unfiltered velocity shows high spectral density only below $50 \mathrm{~Hz}$.

The control law ensures a stable error dynamics if $\dot{V}=S \dot{S} \leq 0$. The optimized control law proposed here is:

$$
U=u_{e q}+u_{1}+u_{2}
$$

The term $u_{e q}$ keeps the system on the sliding surface $S=0$ :

$$
u_{e q}=\frac{A^{\prime} P_{2}}{A}+\frac{\dot{x} \hat{\gamma}}{A}+\frac{m \ddot{x}_{S E T}}{A}+\frac{m\left(\dot{x}_{S E T}-\dot{x}\right) \lambda}{A}+\frac{\hat{F}_{a} \operatorname{sign}(\dot{x})}{A}
$$

The terms $\hat{\gamma}$ and $\hat{F}_{a}$ are the nominal values of viscous and static friction provided by the cylinder manufacturer. The remaining terms in (5) are saturation functions that compensate for model uncertainty:

$$
\begin{aligned}
& u_{1}=a \times \operatorname{sat}(S / b)=\left\{\begin{array}{cc}
a \times \operatorname{sign}(S / b) & |S| \geq b \\
a \times S / b & |S|<b
\end{array}\right. \\
& u_{2}=\left\{\begin{array}{cl}
c \times \operatorname{sat}(S / e) & |S|<b \\
0 & |S| \geq b
\end{array}\right.
\end{aligned}
$$

Assuming known the bounds $\left|\hat{F}_{a}-F_{a}\right| \leq F \quad$ and $|\hat{\gamma}-\gamma| \leq \Gamma$ the positive parameters $a$ and $c$ are chosen as:

$$
\begin{gathered}
a=F / A+\Gamma|\dot{x}| / A+\eta \\
c=F / A+\Gamma|\dot{x}| / A
\end{gathered}
$$

The boundary layer thickness $b$ and $e(e<b)$ and the positive constant $\eta$ are empirically tuned. To verify the stability of the control scheme (5) the derivative $\dot{V}$ is computed differentiating (4) and substituting (3) and (5):

$$
\begin{aligned}
& \dot{V}=\frac{S}{m}\left(\left(F_{a}-\hat{F}_{a}\right) \operatorname{sign}(\dot{x})+(\gamma-\hat{\gamma}) \dot{x}\right)-\frac{S A}{m}\left(u_{1}+u_{2}\right) \\
& =S\left(\Delta f-A u_{1}-A u_{2}\right) / m
\end{aligned}
$$

The term $\Delta f$ in (9) represents the maximum variation of the friction forces. Substituting (7) and (8) we rewrite (9) as:

$$
\dot{V} \leq\left\{\begin{array}{cc}
-\eta|S| \frac{A}{m}<0 ; & |S| \geq b \\
-\left(\frac{F}{m}+\frac{\Gamma|\dot{x}|}{m}+\frac{\eta A}{m}\right)\left(\frac{S^{2}}{b}\right)<0 ; & b>|S|>e \\
S / m\left(\Delta f-(F+\Gamma|\dot{x}|)\left(\frac{S}{b}+\frac{S}{e}\right)-\frac{\eta A S}{b}\right) ; & |S| \leq e
\end{array}\right.
$$

Since $\dot{V} \leq 0$ for $|S|>e$ the system is globally uniformly bounded under the control law (5). For $|S| \geq b$ the control scheme is equivalent to a standard robust SMC. It is apparent that a larger $\eta$ produces a more responsive control action and a faster convergence to the bound $|S| \leq b$. Adding $u_{2}$ inside the boundary layer makes $\dot{V}$ negative definite for $b>|S|>e$ which leads to higher accuracy with the same tuning of $\eta$ and $b$.

For comparison purposes the following baseline control law is considered as an alternative to (5):

$$
\begin{aligned}
& U^{\prime}=u_{e q}+u_{1}^{\prime} \\
& u_{1}^{\prime}=a \times \operatorname{sat}\left(S / b^{\prime}\right) \\
& b^{\prime}=e
\end{aligned}
$$

In this case the system is also bounded by $|S|=e$ however a smaller value of $\eta$ should be chosen to avoid overshoot and oscillations. This also results in less responsive control. In the proposed control scheme (5) instead the contribution of the term $\eta$ decreases linearly for $|S|<b$. At the same time $u_{2}$ balances completely the friction forces for $b>|S|>e$. Consequently a fast convergence to $|S| \leq b$, desirable for tracking tasks, is followed by a more gentle convergence to $|S|=e$, which is desirable for point-to-point positioning tasks. 


\section{EXPERIMENTAL RESULTS}

The experimental tests aimed to prove the MRI compatibility of the prototype and to assess its accuracy in the MR scanner.

\section{A. MRI Compatibility}

The MRI compatibility of the prototype was tested in a 3T MRI scanner (Siemens Verio). The prototype was placed above a cylindrical phantom (Nickel Sulphate solution) and the needle guide was aligned with the scanner isocenter. The imaging sequences used were a Turbo Spin Echo (TSE, TR = $3000 \mathrm{~ms}, \mathrm{TE}=12 \mathrm{~ms}, \mathrm{FA}=180^{\circ}, \mathrm{FOV}=400 \times 400$, Slice Thickness $=5 \mathrm{~mm}$ ) and a True fast imaging with steady-state precession (TrueFISP, TR $=1055 \mathrm{~ms}, \mathrm{TE}=1.57 \mathrm{~ms}, \mathrm{FA}=44^{\circ}$, $\mathrm{FOV}=256 \times 256$, Slice Thickness $=8 \mathrm{~mm}$ ) in conjunction with the scanner Body coil. In total four sets of images were acquired considering first the positioner disconnected from the control unit, then powered on but not moving, and finally with each piston executing a sinusoidal trajectory. The fourth condition used for comparison refers to the phantom alone. The prototype did not produce visible artifacts within the region of interest with any of the sequences used. The variation in signalto-noise ratio (SNR) compared to the phantom alone calculated according to [15] remained below $3 \%$ for all test scenarios (Table II). Additionally no noise in the measured position was detected as a result of the MR environment.

TABLE II

MRI COMPATIBILITY OF THE NEEDLE POSITIONER

\begin{tabular}{|c|c|c|c|c|}
\hline \multirow{2}{*}{ Setup } & \multicolumn{2}{|c|}{ TrueFISP } & \multicolumn{2}{c|}{ TSE } \\
\cline { 2 - 5 } & SNR & \%Variation & SNR & \%Variation \\
\hline $\begin{array}{c}\text { Phantom } \\
\text { (reference) }\end{array}$ & 115.5 & & 546 & \\
\hline Power off & 115.1 & $-0.4 \%$ & 536 & $-1.8 \%$ \\
\hline Power on & 115.2 & $-0.3 \%$ & 531 & $-2.7 \%$ \\
\hline Movement & 114.2 & $-1.1 \%$ & 530 & $-2.9 \%$ \\
\hline
\end{tabular}

Variations in SNR calculated for test in 3T MR scanner.

\section{B. Robot Accuracy}

The advantages of the optimized control scheme (5) are highlighted with a set of step responses on Cylinder 1 alone. Tests with step amplitudes of $0.5 \mathrm{~mm}, 1 \mathrm{~mm}, 5 \mathrm{~mm}, 10 \mathrm{~mm}$ were repeated 10 times and control scheme (5) was compared with the baseline control law (11). The numeric values of controller and model parameters are listed in Table III. The value $b^{\prime}=0.01$ was used in (11) in order to present a meaningful comparison since choosing $b^{\prime}=e$ resulted in excessive chattering. The tests were also performed using a $2.5 \mathrm{~kg}$ mass

TABLE III

CONTROLLER AND MODEL PARAMETERS FOR CYLINDER 1

\begin{tabular}{|c|c|c|c|c|c|c|c|c|}
\hline Parameters & $\lambda$ & $\hat{F_{a}} / A$ & $F / A$ & $\hat{\gamma} / A$ & $\Gamma / A$ & $b$ & $e$ & $\eta$ \\
\hline Value & 12.5 & 0.35 & 0.35 & 20 & 20 & 0.1 & 0.004 & 0.75 \\
\hline unit & $\mathrm{Hz}$ & \multicolumn{2}{|c|}{ bar } & \multicolumn{2}{c|}{ bar s/m } & \multicolumn{2}{|c|}{$\mathrm{m} / \mathrm{s}$} & bar \\
\hline
\end{tabular}

Parameters $b, e, \eta$ are manually tuned; $\lambda$ is chosen as per Section IV.
TABLE IV

STEP RESPONSE FOR CYLINDER

\begin{tabular}{|c|c|c|c|c|}
\hline Controller & \multicolumn{2}{|c|}{$\begin{array}{c}U=u_{e q}+u_{1}+u_{2} \\
\text { Optimized Scheme (5) }\end{array}$} & \multicolumn{2}{|c|}{$\begin{array}{c}U^{\prime}=u_{e q}+u_{1}^{\prime} \\
\text { Baseline Scheme (11) }\end{array}$} \\
\hline Load [kg] & 0 & 2.5 & 0 & 2.5 \\
\hline $\begin{array}{c}\text { Overshoot } \\
\text { [mm] }\end{array}$ & 0.01 & 0 & 1.15 & 1.63 \\
\hline $\begin{array}{c}\text { Settling time } \\
\text { [s] }\end{array}$ & 0.66 & 0.77 & 0.64 & 1.40 \\
\hline $\begin{array}{c}\text { Steady state } \\
\text { error [mm] }\end{array}$ & 0.10 & 0.14 & 0.10 & 0.13 \\
\hline
\end{tabular}

Values are the maximum over a set of 4 step responses with amplitudes $0.5 \mathrm{~mm}, 1 \mathrm{~mm}, 5 \mathrm{~mm}$ and $10 \mathrm{~mm}$ each repeated 10 times.

to evaluate the robustness of the controller. Cylinder 1 was mounted horizontally as in the prototype. Compared to the baseline scheme (11), the optimized controller (5) achieved noticeably lower overshoot and equivalent steady-state error in all test conditions (Table IV) and shorter settling time in the test with the $2.5 \mathrm{~kg}$ mass.

Subsequently the steady-state error of each actuated axis of the needle positioner was evaluated over 100 point-to-point movements with amplitudes varying from $0.3 \mathrm{~mm}$ to $20 \mathrm{~mm}$ in both directions of motion starting from the retracted position. The maximum error for all axes was below $0.16 \mathrm{~mm}$ and the highest mean absolute error was below $0.1 \mathrm{~mm}$. The dynamic response was evaluated actuating the pistons first separately and then simultaneously. At the same time the pressure in the front cylinder chambers was monitored with a sensor (MPX4520GDP, 35 mbar accuracy) on the common supply line. The pistons followed smoothly the prescribed constantjerk trajectories (up to $40 \mathrm{~mm}$ executed in $2 \mathrm{~s}$ ). Moreover no coupling between actuators and no noticeable variation in the measured pressure was detected in spite of the common supply to the front cylinder chambers (Fig. 5).
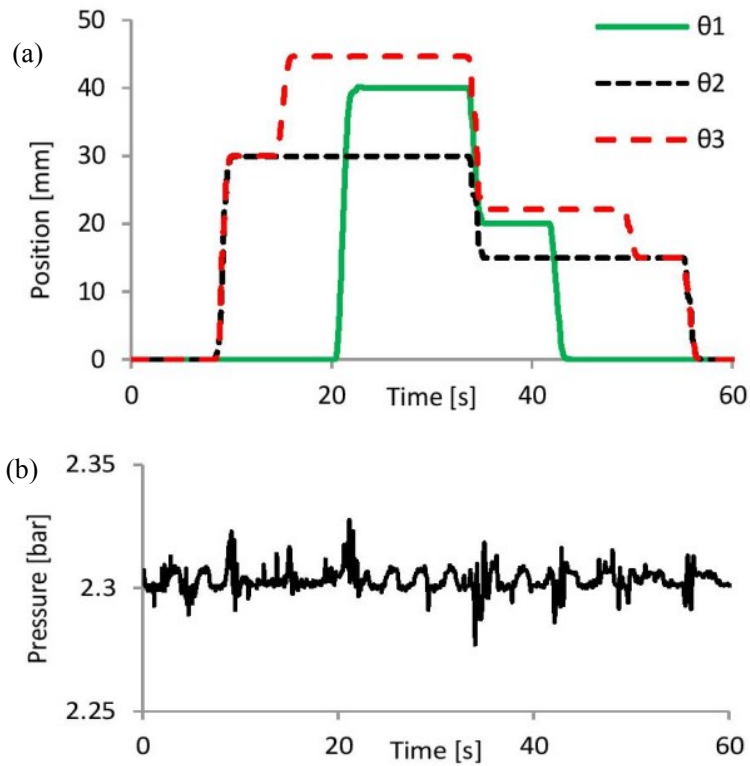

Fig. 5. Constant jerk trajectories for each piston $\left(\theta_{1}, \theta_{2}, \theta_{3}\right)$ with $2 \mathrm{~s}$ set for the movements (a). Pressure in the cylinders' front chambers (b). 


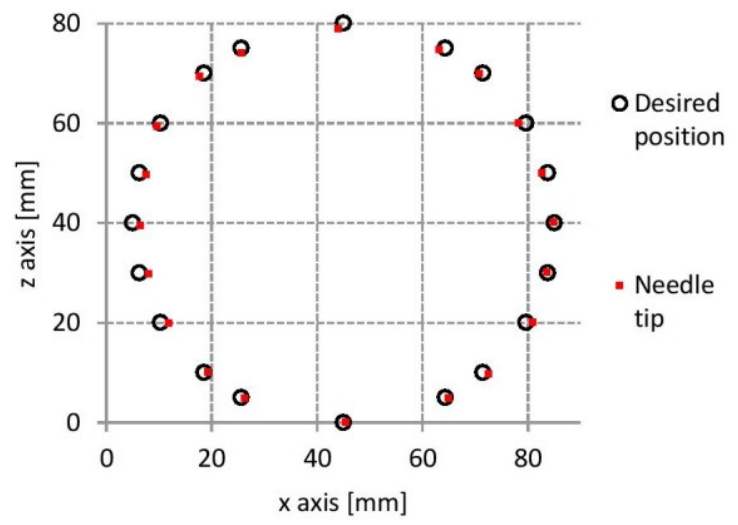

Fig. 6. Needle-tip desired and actual position on the $\boldsymbol{x}_{1}-\boldsymbol{z}_{1}$ plane in the base frame $F 1$ of the positioner.

The accuracy of the positioner was assessed in the MRI scanner over 20 target points within the workspace (Fig. 6). A micro-coil fiducial was placed on an adapter $200 \mathrm{~mm}$ long attached to the needle guide. This setup simulates a fully inserted needle and amplifies position errors of $\theta_{2}$ and $\theta_{3}$ by a factor 5 , corresponding to the ratio between adapter length $l$ and distance $t(1)$. The coordinates of the micro-coil in the reference frame $F 0$ of the MRI scanner were calculated using the tracking algorithm presented in [10]. Initially the reference frame $F 1$ was automatically registered with respect to the scanner frame $F 0$ computing the position of 4 points with (2) and measuring the position of the micro-coil fiducial with the tracking algorithm. Subsequently the desired marker position in the base frame $F 1$ was calculated with (2) from the set values of the joint coordinates and then transformed in the scanner frame $F 0$. The position of the 20 points was measured three times over two hours, which corresponds to the average duration of a clinical procedure. The deviation was calculated as distance between the desired and the measured position. The mean position error for each dataset is between $1 \mathrm{~mm}$ and $1.5 \mathrm{~mm}$ while the maximum error is below $2.1 \mathrm{~mm}$ (Table $\mathrm{V}$ ).

TABLE V

ACCURACY IN 3T MR SCANNER

\begin{tabular}{|c|c|c|c|}
\hline & Dataset 1 & Dataset 2 & Dataset 3 \\
\hline $\begin{array}{c}\text { Mean error } \\
\text { [mm] }\end{array}$ & 1.32 & 1.36 & 1.21 \\
\hline $\begin{array}{c}\text { Maximum } \\
\text { error [mm] }\end{array}$ & 2.07 & 2.08 & 2.06 \\
\hline
\end{tabular}

Needle-tip position error for 3 datasets over 2 hours in 3T MR scanner.

\section{CONCLUSIONS}

The first prototype of a MRI-compatible needle positioner with low pressure pneumatic actuation and long supply lines intended for MRI-guided LITT of the liver has been presented. The prototype can operate inside a high-field MR scanner while being remotely actuated form the control room. The MRI compatibility of the prototype was investigated showing minimum alterations to the MR images. An optimized SMC scheme allowed achieving sufficient point-to-point accuracy with smaller overshoot and shorter settling time compared to a standard SMC design. Experiments in the MR scanner suggest that the accuracy of the system would be sufficient for clinical use. Further work will include the development of a second prototype with an additional angular DOF to allow inclined needle insertions on both sagittal and transversal planes. We also aim at further enhancing the accuracy of the prototype in order to offer clear advantages over the manual procedure.

\section{ACKNOWLEDGMENT}

The support of the i4i Grant II-AR-1109-11011 is gratefully acknowledged.

\section{REFERENCES}

[1] Gough-Palmer, A.L.; Gedroyc, W.M. "Laser ablation of hepatocellular carcinoma--a review," World J Gastroenterol. 2008.

[2] Dick, E.A.; Taylor-Robinson ,S.D.; Thomas, H.C.; Gedroyc, W.M.."Ablative therapy for liver tumours," Gut. May 2002.

[3] Vogl, T.J.; Straub, R.; Eichler, .K; Woitaschek, .D;Mack, M.G., "Malignant liver tumors treated with MR imaging-guided laser-induced thermotherapy: technique and prospective results," Radiology,1995.

[4] Gedroyc, W.M., "Magnetic resonance guidance of thermal ablation," Top Magn Reson Imaging. Oct 2005.

[5] N. Hata, R. Hashimoto, and J. Tokuda, "Needle guiding robot for MRguided microwave thermotherapy of liver tumor using motorized remote-center-of-motion constraint," Robotics and Automation (ICRA), 2005 IEEE International Conference on.

[6] Sang-Eun Song; Cho, N.B.; Fischer, G.; Hata, N.; Tempany, C.; Fichtinger, G.; Iordachita, I., "Development of a pneumatic robot for MRI-guided transperineal prostate biopsy and brachytherapy: New approaches," Robotics and Automation (ICRA), 2010 IEEE International Conference on, vol., no., pp.2580,2585, 3-7 May 2010.

[7] Melzer, A.; Gutmann, B.; Remmele, T.; Wolf, R.; Lukoscheck, A.; Bock, M.; Bardenheuer, H.; Fischer, H., "INNOMOTION for Percutaneous Image-Guided Interventions," Engineering in Medicine and Biology Magazine, IEEE, vol.27, no.3, pp.66,73, May-June 2008.

[8] Bo Yang; U-Xuan Tan; McMillan, A.; Gullapalli, R.; Desai, J.P., "Design and implementation of a pneumatically-actuated robot for breast biopsy under continuous MRI," Robotics and Automation (ICRA), 2011 IEEE International Conference on , vol., no., pp.674,679, 9-13 May 2011.

[9] Bo Yang; U-Xuan Tan; McMillan, A.B.; Gullapalli, R.; Desai, J.P., "Design and Control of a 1-DOF MRI-Compatible Pneumatically Actuated Robot With Long Transmission Lines," Mechatronics, IEEE/ASME Transactions on, vol.16, no.6, pp.1040,1048, Dec. 2011.

[10] Brujic, D.; Galassi, F.; Rea, M.; Ristic, M., "A novel algorithm for fast $3 \mathrm{D}$ localization of $\mathrm{N}$ fiducial markers from 1D projections", ISMRM 2012, Melbourne.

[11] Kawamura, D. M., Diagnostic Medical Sonography: Abdomen and Superficial Structures. Philadelphia: Lippincott Williams \& Wilkins, 1997.

[12] Mooring, B. W.; Roth, Z. S.; Driels, M. R., Fundamentals of Manipulator Calibration. New York: Wiley Interscience, 1991. p.165191.

[13] Rea, M.; McRobbie, D.; Elhawary, H.; Tse, Z.; Lamperth, M.;Young, I., "Sub-pixel localisation of passive micro-coil fiducial markers in interventional MRI," Magnetic Resonance Materials in Physics,

[14] Slotine, J.-J, Li, W., Applied Nonlinear Control, 1991: Prentice-Hall.

[15] "Determination of signal-to-noise ratio (SNR) in diagnostic magnetic resonance imaging”, 2008, NEMA Standard Publication MS 1-2008. 